\title{
Reaproveitamento de resíduo de placas de circuito impresso como cargas em compósitos de polipropileno
}

\author{
Reuse of waste printed circuit boards as fillers in \\ polypropylene composites
}

\author{
Eduardo Luis Schneider ${ }^{1}$, Guilherme Dias Grassi ${ }^{1}$, \\ Sandro Campos Amico ${ }^{1}$, Rodrigo de Andrade Chaves ${ }^{2}$, \\ Daniel Claro Mazzuca ${ }^{2}$, Luiz Carlos Robinson².
}

\author{
${ }^{1}$ Departamento de Engenharia de Materiais. Universidade Federal do Rio Grande do Sul - UFRGS, Farroupilha, Rio Gran- \\ de do Sul, RS, Brasil. \\ ${ }^{2}$ Universidade Feevale, Novo Hamburgo, Rio Grande do Sul, RS, Brasil. \\ e-mail: prof.eduardo@ufrgs.br, guilhermegrassi@hotmail.com, amico@ufrgs.br, rodrigoch@live.com, danielclaromazzu- \\ ca@gmail.com, luiz.robinson@feevale.br
}

\begin{abstract}
RESUMO
As placas de circuito impresso (PCIs) são as partes mais valiosas dos resíduos eletrônicos, pois possuem uma considerável quantidade de metais com potencial de recuperação. Processos de reciclagem com o intuito de recuperar metais de PCIs têm despertado interesse ultimamente. Contudo, uma fração destes resíduos não é completamente aproveitada e tem destino incerto, como ocorre para a fração não-metálica e o pó gerados durante os processos de recuperação de metais. $\mathrm{O}$ foco deste estudo foi o emprego do pó gerado nos processos de cominuição mecânica, separação granulométrica, magnética e eletrostática de PCIs controladoras e indicadoras de temperatura de câmaras frias, para obter compósitos de matriz de polipropileno (PP) com diferentes teores de pó de PCIs, 5, 10 e 20\% em massa. Os materiais foram pesados, misturados, moldados por injeção e posteriormente caracterizados por ensaios de tração, dureza Shore D, espectroscopia de infravermelho, análise termogravimétrica e microscopia eletrônica de varredura. Os resultados dos ensaios de tração e dureza indicaram propriedades levemente superiores às do PP puro, exceto para a deformação na ruptura, que foi reduzido em até 2,7 vezes para a amostra com $20 \%$ de resíduo. O desenvolvimento de compósitos utilizando o pó de PCIs se mostrou viável em aplicações onde as propriedades resistência à tração, rigidez e dureza são determinantes, mesmo para um elevado teor de resíduo (20\%) agregado.
\end{abstract}

Palavras-chave: Placas de circuito impresso. Resíduos eletrônicos. Compósitos. Reciclagem.

\begin{abstract}
Printed Circuit Boards (PCBs) are the most valuable parts of e-waste because they have a considerable amount of metals with recovery potential. Recycling processes aimed at recovering metals from PCBs have aroused great interest recently. However, there is a fraction of this waste that is not utilized and has uncertain destination, e.g. the non-metallic fraction and the dust generated during metal recovery processes. The focus of this study was to employ the powder generated in the mechanical comminution, granulometric, magnetic and electrostatic separation processes of controller and temperature indicator PCBs of cold rooms to obtain polypropylene (PP) matrix composites with distinct PCBs powder content, 5, 10 and $20 \%$ in weight. The sample was collected during the mechanical comminution processes. The materials were weighed, mixed, injection molded and later characterized using tensile tests, Shore D hardness, infrared spectroscopy, thermogravimetric analysis and scanning electron microscopy. Results of the tensile and hardness tests showed properties slightly superior to pure PP, except for strain at break, which was reduced up to 2.7 times for the sample with $20 \%$ of residue. In all, the development of composites using the powder of PCBs was considered feasible for applications where tensile strength, stiffness and hardness are important, even for a large amount of residue added (20\%).
\end{abstract}

Keywords: Printed circuit boards. E-waste. Composites. Recycling. 


\section{INTRODUÇÃO}

Equipamentos elétricos e eletrônicos (REEE ou e-waste) têm uma curta vida útil devido aos fatores como o avanço tecnológico, a obsolescência programada e o consumismo. No ano de 2016, a geração global de lixo eletrônico atingiu 44,7 milhões de toneladas, equivalente $6,1 \mathrm{~kg}$ por habitante, com previsão de atingir 52,2 milhões de toneladas e $6,8 \mathrm{~kg}$ por habitante em 2021 [1]. A reciclagem, por exemplo, da fração de polímeros desse e-waste depende da correta separação na desmontagem bem como do controle do processamento [2].

As placas de circuito impresso (PCIs) são componentes essenciais utilizados na fabricação de produtos eletrônicos, desde os mais simples eletrodomésticos até máquinas industriais mais avançadas. Em sua composição, há vários materiais passíveis de recuperação e reutilização, tendo os metais uma maior rentabilidade. Metais valiosos que podem ser recuperados incluem cobre, ouro, prata e paládio, com pureza e concentração superiores as da mineração convencional.

O processamento de PCIs para recuperação de metais valiosos utiliza uma combinação de processos físico-mecânicos e químicos. O processamento pode ser em geral dividido em três etapas: desmanche, trituração e separação. Segundo LI et al. 2017 [3], a primeira etapa é fundamental para separar partes grandes e de fácil identificação. O processo de desmanche de diferentes componentes e materiais do e-waste é descrito por GHOSH et al. 2015 [4] como uma das principais etapas do processo de reciclagem, onde devem ser separados materiais perigosos, como baterias e capacitores, e o que for possível de identificar e desmontar facilmente, como invólucros plásticos, vidros e partes metálicas. Essa etapa facilita a separação dos diferentes materiais presentes e evita que componentes perigosos entrem no processo de trituração. Em contrapartida, KUMARA et al. 2017 [5] relatam alguns pontos que devem ser observados para que não haja perda de produtividade no processo, como a dificuldade em desmontar novas e complexas tecnologias, o tempo consumido e o aumento do risco de contaminação humana por metais pesados. Tais fatores devem ser levados em consideração ao definir quais as partes são vantajosas ou não de serem desmontadas.

A etapa de trituração tem a função de desprender, através da quebra dos resíduos, os diferentes materiais que estão unidos aos equipamentos, seja por solda, cola ou parafusos (ZENGA et al. 2012 [6]). KUMARA et al. 2017 [5] destacam fatores negativos também nesta etapa, como a alta geração de poeira, a perda em massa de até $40 \%$ devido à geração de pó e o aumento no investimento com maquinário.

Os métodos de separação do material triturado são baseados, em geral, em três diferentes princípios: magnético, físico e eletrostático. A separação magnética visa separar a parcela ferromagnética do restante da amostra. Sabe-se que os metais ferrosos presentes no $e$-waste não possuem um valor agregado muito alto, então o foco se concentra principalmente na identificação da parcela metálica não-magnética. Dentre os métodos físicos, propriedades como tamanho da partícula e densidade são utilizados para separar os diferentes materiais. KUMAR et al. 2015 [7] descrevem o processo de separação pneumática da amostra em uma fração mais densa e outra menos densa utilizando um fluxo de ar. KOVACEVIC et al. 2017 [8] utiliza um sistema com peneiras vibratórias para classificar o material de acordo com a granulometria, e ESTRADA-RUIZ et al. 2016 [9] apresentam um sistema de flutuação inversa para separar as frações metálica e não-metálica com base na densidade do material.

O princípio eletrostático se baseia no uso do efeito eletrostático de alta tensão para identificar materiais condutores e não-condutores e, quando aplicado após a separação magnética, pode-se identificar o restante dos metais presentes na amostra, que apresentam maior valor agregado (ROCCHETTI et al. 2018 [10]). Há três técnicas típicas de separação por condutividade elétrica: separação por corrente de Foucault, separação eletrostática corona e separação triboelétrica. Na separação por corrente de Foucault, utilizam-se separadores de correntes de Eddy, que são equipamentos que permitem o trabalho contínuo separando metais não ferrosos, como alumínio, latão, bronze, entre outros, de outros materiais. Separação eletrostática corona é uma técnica importante utilizada para partículas finas com faixa de tamanho de 0,6 a $1.2 \mathrm{~mm}$ a partir da exploração do efeito corona e utilização de um sistema de eletrodos que são determinados pelos parâmetros: velocidade do rotor, teor de umidade e o tamanho das partículas que têm o maior efeito na determinação dos resultados da separação. Separação triboelétrica é realizada por um separador de correia triboelétrico que realiza o processo de eletrização por atrito dos materiais dos PCIs que pertencem a série triboeletrica. O processo mais comum para esse fim é o da descarga Corona, descrito por LI et al. 2007 [11], em que é gerado um campo eletrostático de alta tensão por um eletrodo de corona e um eletrodo adicional. As forças do campo atuam de forma diferente nas partículas metálicas e não-metálicas, possibilitando a separação.

Nos diferentes estágios físico-mecânicos, é gerada uma grande quantidade de pó que se dispersa facilmente na atmosfera e se deposita nas máquinas. Plantas mais desenvolvidas possuem sistemas próprios para coleta e destinação do pó, porém o processo de separação e recuperação de metais valiosos a partir deste pó é mais complexo em relação às partículas de maior granulometria. Considerando-se que apenas uma porcentagem da poeira gerada durante o processamento mecânico de PCIs pode ser reutilizada, sendo a outra parcela descartada em aterros, caracteriza-se então um problema sério na destinação do lixo eletrônico, pois este contém substâncias tóxicas como mercúrio, cádmio e chumbo (SCHNEIDER et al. $2018[2])$. 
A utilização de pó de PCIs como reforço para produção de compósitos de polipropileno (PP) permite substituir materiais tradicionalmente utilizados como fibra de vidro, talco e mica. Estes tipos de compósito são empregados em aplicações, como cadeiras plásticas, lixeiras, invólucros de eletrodomésticos, cases de baterias, caixas para armazenamentos em geral, onde é necessário aumentar a resistência mecânica e módulo de elasticidade do PP puro. Zheng et al. 2009 [12] verificaram que os não-metais reciclados, a partir de PCIs podem aumentar a resistência a flexão com adições de até $30 \%$. Além disso, atribuíram esta melhoria mecânica obtida nos compósitos de PP devido as partículas dispersas que podem levar a formação de microfissuras e evitar que as fissuras maiores, como as que ocorrem no PP puro, sejam formadas. Yang et al. 2015 [13] estudaram a obtenção de pós de PCIs empregando diferentes métodos de moagem (em panela, a jato e planetária de esferas) para produção de compósitos de PP e verificaram que a dispersão do pó e a adesão interfacial com a matriz de polipropileno foram muito melhores por moagem em panela, conferindo as melhores propriedades mecânicas do que outros métodos de moagem.

Assim, o foco do presente estudo foi a obtenção e o emprego do pó gerado nos processos de cominuição mecânica e separação granulométrica, magnética e eletrostática de PCIs de controladoras e indicadoras de temperatura de câmaras frias, empregando-o para obter compósitos de matriz de PP com diferentes teores de pó de PCIs e caracterizar as amostras através de espectroscopia de infravermelho, análise termogravimétrica e microscopia eletrônica de varredura, bem como avaliar as propriedades mecânicas por ensaios de tração e dureza Shore D.

\section{MATERIAIS E MÉTODOS}

\subsection{Obtenção do pó de $\mathrm{PCl}$ e do compósito com PP}

Nessa pesquisa, foi realizado o processamento de aproximadamente $13 \mathrm{~kg}$ de PCIs doados pela empresa Reverse - Gestão de Resíduos, localizada em Novo Hamburgo - RS, gerando um resíduo na condição de pó. A Figura 1 ilustra o fluxograma do processamento de PCIs empregado para recuperação de metais valiosos e o pó gerado oriundo da fração não metálica que foi utilizado neste trabalho. O processo apresenta todas as etapas descritas anteriormente. $\mathrm{O}$ desmanche se referiu à simples retirada mecânica de componentes indesejáveis, ou seja, nesta fase devem ser separados os materiais perigosos, como baterias e capacitores, e o que for possível de identificar e desmontar facilmente, como invólucros plásticos, vidros e partes metálicas. Essa etapa facilita a separação dos diferentes materiais presentes nos REEE e evita que componentes perigosos entrem no processo de trituração e prejudiquem sua eficiência. Já a fase de trituração foi feita em três partes (trituração, moagem até $4 \mathrm{~mm}$ e moagem até $2 \mathrm{~mm}$ ) ou seja, na parte da trituração, o objetivo foi retirar os metais das placas de base das PCIs usadas, porque as PCIs residuais são compostos de resina reforçada e peças metálicas, como fios e articulações que têm uma alta dureza e tenacidade, assim, frequentemente, é necessário o esmagamento que é posterior à trituração primária em duas etapas (moagem até $4 \mathrm{~mm}$ e moagem até $2 \mathrm{~mm}$ ) para uma adequada triagem que não foi usada apenas para preparar uma alimentação de tamanho uniforme a determinado processo mecânico, mas também para atualizar o conteúdo de metais. A triagem é necessária porque o tamanho das partículas e as propriedades de forma dos metais são diferentes das propriedades dos plásticos e cerâmicas. Então, todo o método de trituração na recuperação de metais usa a tela rotativa, ou trammel, que é uma unidade amplamente usada na sucata de automóveis e no processamento de resíduos sólidos. Já a etapa de separação incluiu separação magnética, separação granulométrica e separação eletrostática.

Na separação magnética, foram utilizados separadores de tambor de baixa intensidade, que são amplamente utilizados para a recuperação de metais ferromagnéticos de metais não ferrosos e outros resíduos não magnéticos. O desenvolvimento do compósito se iniciou pela definição do teor das fases constituintes. Estudos realizados por WANG et al. 2010 [14], MUNIYANDI et al. 2013 [15] e ZHENG et al. 2009 [16], que utilizaram como matriz do compósito policloreto de vinila (PVC), polietileno de alta densidade (PEAD) e polipropileno (PP), respectivamente, indicaram um teor de até $30 \%$ de resíduo em suas análises. Com base nesses dados, foi definida a composição de 4 amostras utilizando 5\%, 10\%, 20\% e $30 \%$ de resíduo de PCIs, além de uma amostra de PP puro. 


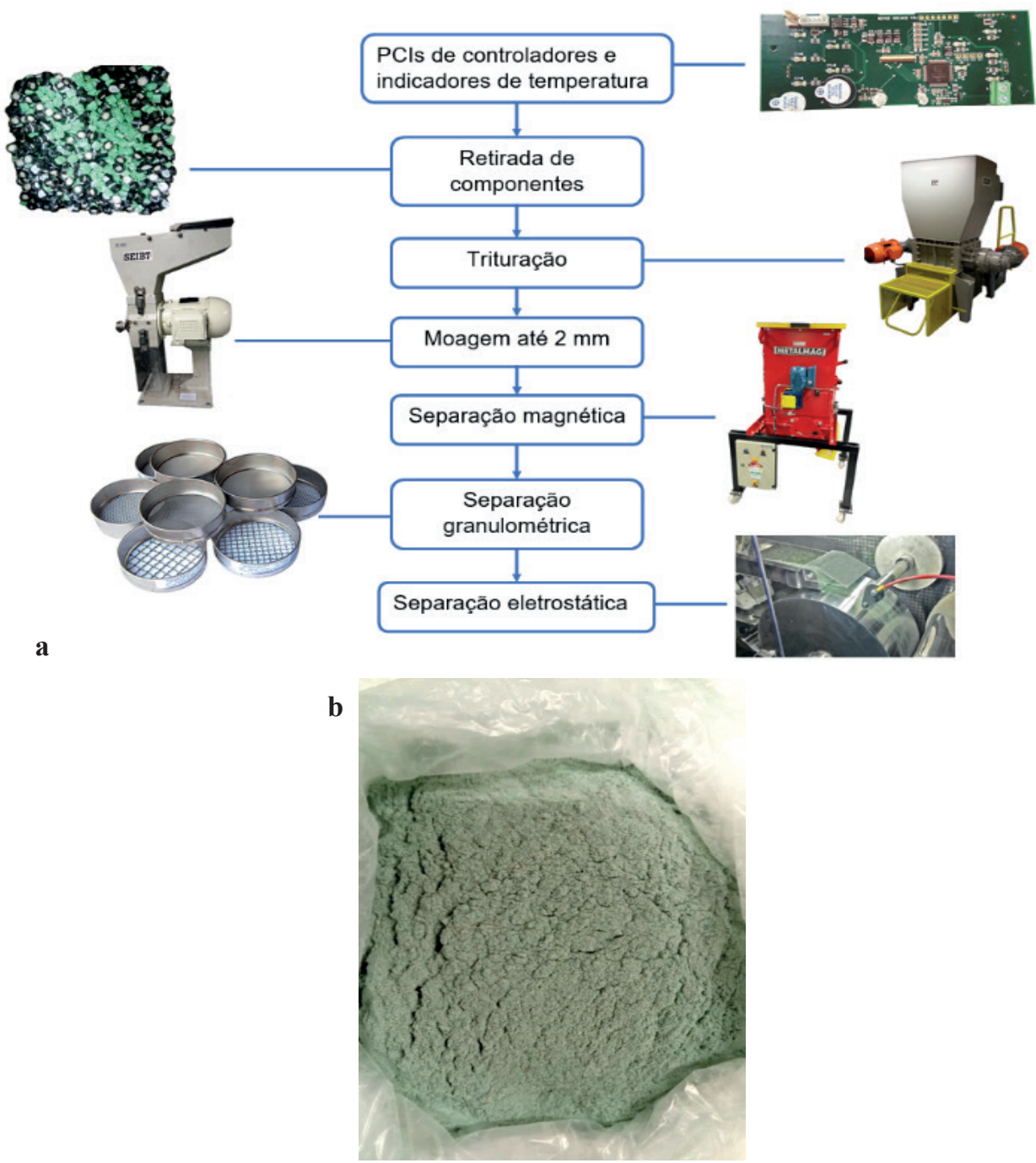

Figura 1: (a) Etapas do processamento de placas PCIs para recuperação de metais valiosos e (b) resíduo de PCIs em pó obtido. Fonte: Os autores.

A obtenção da massa dos materiais para a composição das formulações descritas foi realizada com uma balança semi-analítica marca Marte modelo AS 5000C. Um recipiente no formato de jarra foi utilizado para acomodar o polipropileno virgem e o resíduo durante a pesagem e a mistura, procedimentos realizados manualmente. Para produzir os corpos de prova, foi utilizada uma máquina injetora modelo 140, da marca Bonmaq, com capacidade para dosagem de $120 \mathrm{~cm}^{3} \cdot \mathrm{s}^{-1}$, pressão de injeção de até 400 bar e 4 zonas de aquecimento. O polipropileno utilizado foi H502HC em formato de pellets fornecido pela empresa Braskem. De acordo com o fabricante, trata-se de um homopolímero de baixo índice de fluidez projetado para aplicações que necessitam de elevada rigidez/tenacidade e boa processabilidade.

As formulações foram injetadas em um molde de alumínio com cavidades em formato de corpos de prova tipo IV da norma ASTM D638, para ensaio de tração de polímeros. Foram realizados testes preliminares para definição do perfil de temperaturas adotado. Foi constatado que a inclusão de resíduo estava contribuindo para o decréscimo da fluidez do material, ocasionando o preenchimento incompleto do molde para uma temperatura no bico injetor (zona 4) de $180{ }^{\circ} \mathrm{C}$. Por isso, elevou-se esta temperatura para $190^{\circ} \mathrm{C}$, o que possibilitou a injeção com preenchimento total do molde. Assim, o perfil de temperatura adotado para a injeção dos corpos de prova foi: $160 \pm 5^{\circ} \mathrm{C}, 170 \pm 5{ }^{\circ} \mathrm{C}, 180 \pm 5^{\circ} \mathrm{C}$ e $190 \pm 5{ }^{\circ} \mathrm{C}$ para a temperatura da zona 1, zona 2, zona 3 e zona 4 (bico injetor), respectivamente.

Nesta etapa de injeção, foi constatada a impossibilidade de processamento da amostra PP_PCI30 devido ao entupimento da injetora. Isto pode ter ocorrido pela redução crítica da fluidez do material com a adição deste elevado teor de carga, visto que o PP H502HC puro já é um material de baixa fluidez. Também pode ter ocorrido uma má homogeneização da mistura PP/PCI, gerando um acúmulo local de resíduo. Outra possibilidade é a inadequação do equipamento de realizar este procedimento devido a fatores como capacidade da máquina ou tipo de rosca injetora. Dificuldade similar foi relatada por MUNIYANDI et al. 2013 [15] processando compósitos de PEAD com 30\% do resíduo de PCIs. 


\subsection{Caracterização do resíduo e dos compósitos obtidos}

O resíduo de PCIs foi analisado, antes de ser agregado ao compósito, por separação granulométrica, espectroscopia de infravermelho (FTIR) e análise termogravimétrica (TGA). A separação granulométrica foi realizada com o auxílio de um agitador eletromagnético da marca Bertel, com capacidade para seis peneiras. $\mathrm{O}$ ensaio foi executado com agitação na frequência de $8 \mathrm{~Hz}$ pelo tempo de 10 minutos, repetido por quatro vezes e obtida a média entre os quatro ensaios.

A matéria-prima virgem PP foi analisada por espectroscopia de infravermelho e TGA. Os corpos-de prova injetados de PP puro e dos compósitos PP com PCIs foram analisados em ensaios de tração, dureza, TGA e espectroscopia de infravermelho, além da análise em microscópio eletrônico de varredura (MEV).

Os ensaios de tração foram realizados conforme a norma ASTM D638/03 em dinamômetro da marca MAQtest com célula de carga de $200 \mathrm{kgf}$. O ensaio com velocidade de deslocamento constante de $100 \mathrm{~mm} / \mathrm{min}$. Foram ensaiados cinco corpos de prova de cada formulação, mostrando-se os resultados médios. Para o ensaio de dureza Shore D, utilizou-se um durômetro da marca Mainard modelo M-702A com dispositivo para aplicação de carga controlada e leitura do valor $3 \mathrm{~s}$ após a penetração da agulha. O ensaio foi realizado seguindo a norma DIN 53505, com a medição em nove pontos de cada um de cinco corpos de prova para cada formulação sendo o resultado, o valor reportado referente à media dos pontos.

Para a análise por TGA, foi utilizado o equipamento TGA-51H, da marca Shimadzu. Os parâmetros do ensaio incluíram: rampa de temperatura de 25 a $800^{\circ} \mathrm{C}$, taxa de aquecimento de $10^{\circ} \mathrm{C} / \mathrm{min}$, nitrogênio como gás de arraste a uma vazão de $50 \mathrm{~mL} / \mathrm{min}$. A análise por FTIR foi realizada em espectrômetro, modelo Spectrum Two, da marca PerkimElmer na faixa de número de onda de 400 a $4000 \mathrm{~cm}^{-1}$.

As amostras sofreram fratura criogênica para a análise morfológica no MEV. Após, uma fina camada de ouro foi adicionada sobre a fratura utilizando metalizador da marca Denton Vancuum, modelo Desk V. O MEV utilizado foi de modelo JSM 6510LV da marca Jeol, com intensidade do feixe de elétrons de $10 \mathrm{kV}$, operando com magnificação de $1000 \mathrm{X}$.

\section{RESULTADOS E DISCUSSÕES}

\subsection{Caracterização quanto à granulometria do resíduo de $\mathrm{PCls}$}

A partir da caracterização granulométrica do resíduo de PCIs, foi possível identificar pela média de quatro ensaios, que a amostra é composta por partículas na faixa de: 36\% com até 80 Mesh, 26\% de 80-170 Mesh, 23\% de 170-200 Mesh, 13\% de 200-250 Mesh, 2\% de 250-325 Mesh e 0,1\% de 325-500 Mesh.

O emprego de uma faixa granulométrica mais fina que aproveite uma quantidade maior do pó gerado no processamento das PCI's permite melhor incorporação das partículas pela matriz e mais homogênea fica a superfície do material processado, bem como evitar o desperdício. Por outro lado, esta distribuição de granulometria com maior quantidade na faixa até 80 Mesh tende apresentar maior a resistência mecânica de acordo com ZHENG et al. 2009 [14]. Este estudo descreve a utilização de resíduo não-metálico de PCIs em compósitos com PP e apresenta a inclusão de 10, 20 e 30\% de resíduo de 25, 80 e 150 Mesh na composição das amostras. Estes relataram acréscimo de resistência à tração nas três amostras, e o material com partículas de 80 Mesh obteve melhor resultado, aumentando de forma constante com a inclusão de 10, 20 e $30 \%$ de resíduo. Para as amostras de 25 e 150 Mesh com 30\% de resíduo, no entanto, houve estabilização ou decréscimo na resistência. Portanto, a faixa de percentagens utilizadas de resíduo não-metálico de PCIs é: $5 \%, 10 \%$ e $20 \%$ em massa com granulometria de 80 Mesh.

WANG et al. 2010 [15] relatam a utilização de resíduo não-metálico de PCIs de 60 e 180 Mesh em compósito utilizando PVC, com formulações com 10, 20 e 30\% de resíduo. Os autores obtiveram aumento na resistência à tração em ambas as amostras com a inclusão de $10 \%$ de resíduo, que se manteve praticamente constante com $20 \%$ de resíduo e que reduziu para a amostra de 30\%. MUNIYANDI et al. 2013 [16] usaram PEAD e resíduo não-metálico de PCIs no compósito. Neste estudo, o resíduo era composto pela seguinte granulometria equivalente, 50\% com $100 \mathrm{Mesh}, 25 \%$ com $170 \mathrm{Mesh}$, 14,3\% com 200 Mesh e 10,7\% inferior a isto. Os resultados mostraram uma redução na resistência à tração em relação ao PEAD puro, para as três formulações (10, 20 e 30\% de resíduo).

\subsection{Análise Termogravimétrica}

A Figura 2 apresenta a curva termogravimétrica TG do resíduo de PCIs usado como carga no desenvolvimento do compósito, na qual pode ser observada sua degradação com o acréscimo da temperatura. A curva indica a degradação do resíduo ocorrendo entre 310 e $425^{\circ} \mathrm{C}$, com uma baixa perda de massa de $14,28 \%$ já que foi usado um meio nitretante. Este resultado é coerente com o relatado por SHIN et al. 2019 [17] que, em analise por TGA do resíduo de PCIs encontrou uma faixa de 
degradação entre $\approx 310-470{ }^{\circ} \mathrm{C}$, com perda de massa de cerca de $30 \%$.

Embora o resíduo contenha uma mistura de materiais, foi identificada apenas uma curva TG nessa faixa de temperatura, o que indica uma concentração predominante de um deles. Considerando que a fibra de vidro é um material encontrado em grande proporção nas PCIs ( $\approx 50 \%$ do resíduo) e que as frações não-metálicas desse resíduos contêm $\approx 50-70 \%$ de fibra de vidro, como descrito por ZHENG et al. 2009 [14], sugere-se que seja este o elemento identificado como resíduo no ensaio de TGA.

A curva TG do PP puro também pode ser vista na Figura 2. Foi identificada a degradação entre 444 e $545{ }^{\circ} \mathrm{C}$, com perda de massa de $\approx 96 \%$. MAT-SHAYUTI et al. 2016 [18] indicam em seu estudo o intervalo de degradação do polipropileno entre 283 e $483{ }^{\circ} \mathrm{C}$. A comparação entre matérias-primas virgens é dificultada devido aos diferentes produtores e às diferentes características de um mesmo material. O PP utilizado neste estudo foi o H502HC, enquanto o PP utilizado por MAT-SHAYUTI et al. 2016 [18] foi identificado como G425, sendo produzido pela empresa Polypropylene Malaysia.

A Figura 2 mostra as curvas TG das amostras PP_PCI5, PP_PCI10, e PP_PCI20. Para o PP_PCI5, foi identificado um intervalo de degradação entre $408-535^{\circ} \mathrm{C}$, sendo similar ao observado para o PP puro e não foi identificado resíduo. A ausência do resíduo se deve ao baixo teor de resíduo de PCIs incorporado e pelo tamanho reduzido da amostra (em mg), que não possuía uma parcela detectável do mesmo.

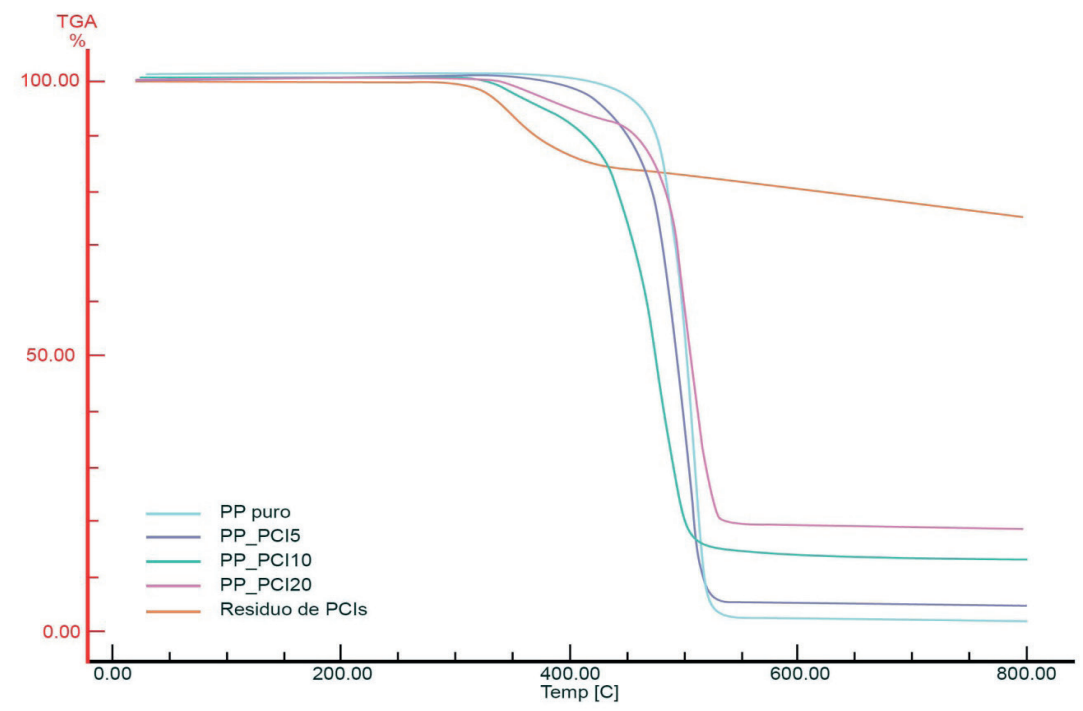

Figura 2: Curvas TG das amostras de resíduo de PCI, PP puro, PP_PCI5, PP_PCI10 e PP_PCI20.

Para as amostras PP_PCI10 e PP_PCI20, como se vê na Figura 3 com as derivadas das perdas de massa, foram observados dois intervalos de degradação que pertencem ao resíduo de PCIs e ao polipropileno, respectivamente. Para o PP_PCI10, o primeiro intervalo ocorre entre $313-388^{\circ} \mathrm{C}$ e o segundo entre $389-520^{\circ} \mathrm{C}$, similar ao apresentado acima para o PP puro. O material remanescente final neste caso se deve à fibra de vidro. Para a amostra PP_PCI20, esses intervalos foram entre $319-389^{\circ} \mathrm{C}$, e entre $427-547^{\circ} \mathrm{C}$, respectivamente. Esses resultados indicam que com o aumento da adição desta carga o compósito tende a se decompor em uma faixa de temperatura maior, indicando um aumento na temperatura máxima de trabalho em relação ao PP puro. 


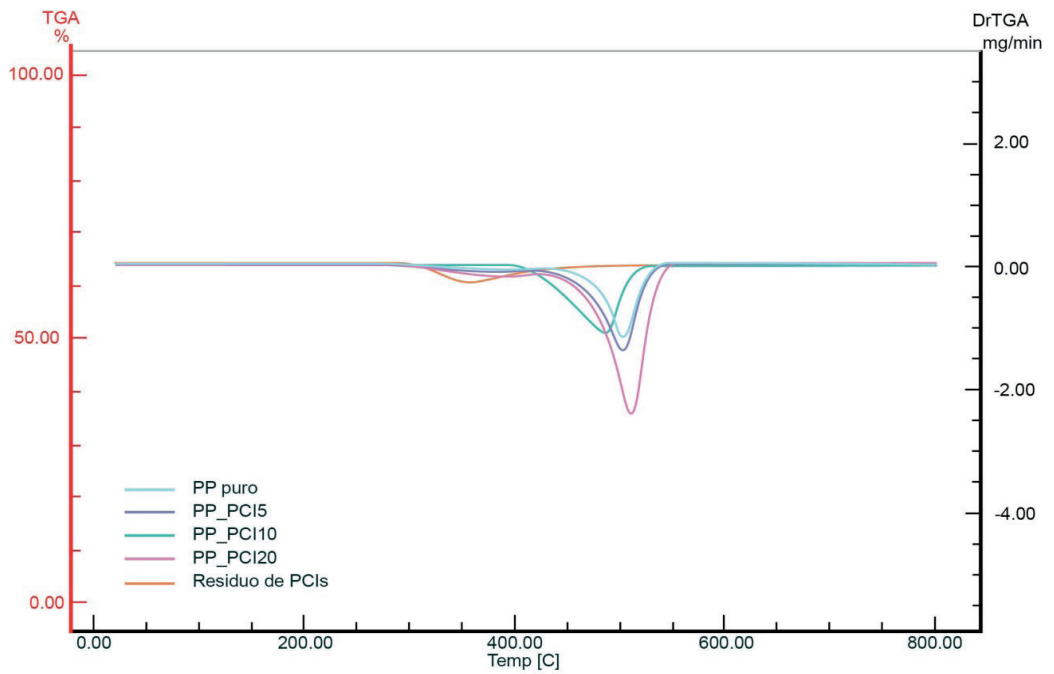

Figura 3: Curvas DTG das amostras de resíduo de PCI, PP puro, PP_PCI5, PP_PCI10 e PP_PCI20.

\subsection{Espectroscopia de Infravermelho}

A Figura 4 apresenta os resultados do espectro de absorção no infravermelho do resíduo utilizado como carga, do PP puro e das amostras PP_PCI5, PP_PCI10 e PP_PCI20 sobrepostos. Praticamente não houve diferença entre os resultados do PP puro e das amostras com adição do resíduo, o que pode estar novamente associado ao baixo teor de PCIs nas amostras ensaiadas e à superposição de alguns picos do PP com do PCIs.

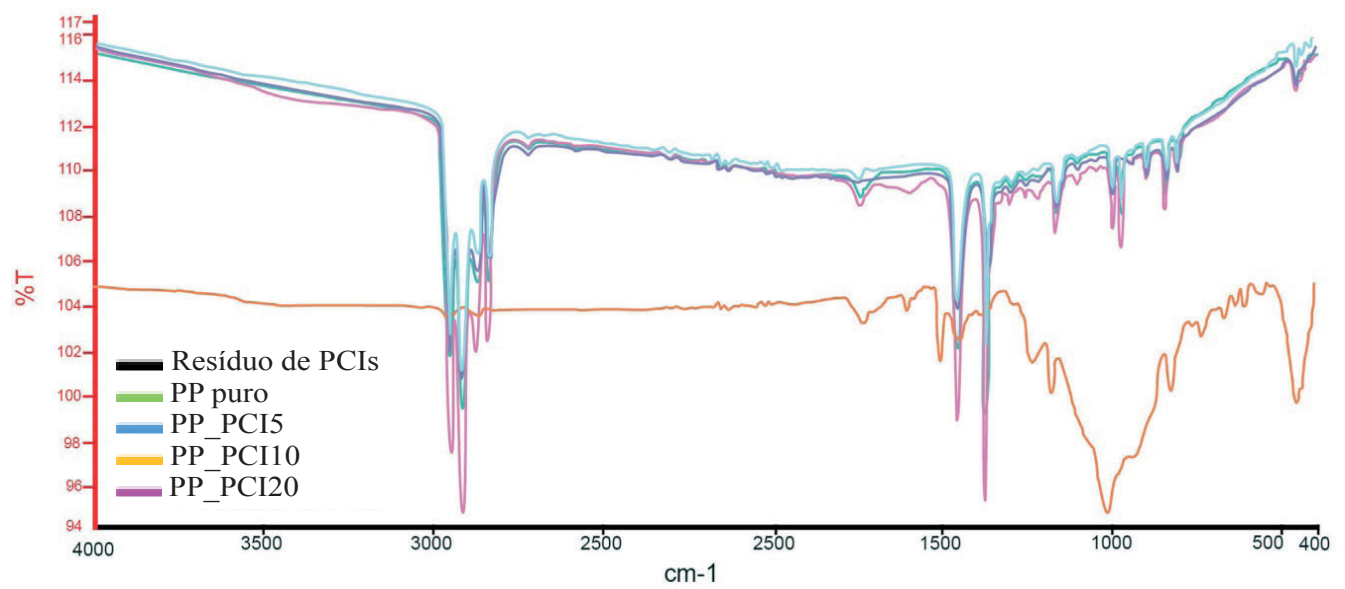

Figura 4: Espectros de infravermelho do resíduo de PCIs, PP puro, PP_PCI5, PP_PCI10 e PP_PCI20.

\subsection{Ensaio de Tração}

O aspecto visual dos compósitos produzidos pode ser observado na Figura 5, com os diferentes corpos de prova injetados. É possível observar um aumento gradativo da cor verde entre as amostras PP puro, PP_PCI5, PP_PCI10 e PP_PCI20, sendo as duas últimas mais similares. $\mathrm{O}$ tom esverdeado que é visto nos corpos de prova é devido à cor verde-escura da maioria das placas de circuito impresso fabricadas. 


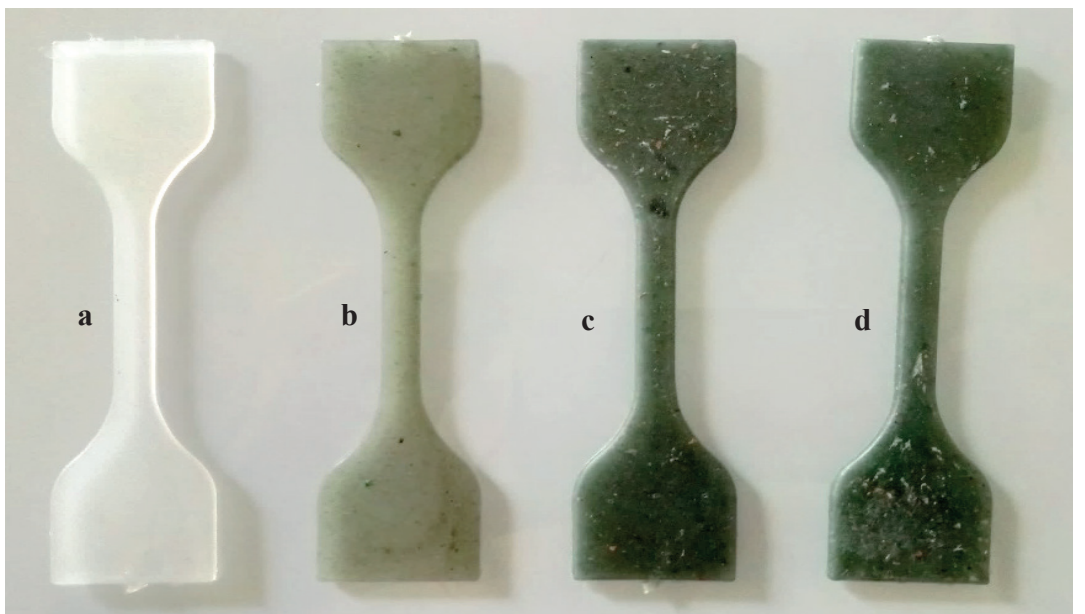

Figura 5: Corpos de prova injetados para ensaio de tração: (a) PP puro; (b) PP_PCI5; (c) PP_PCI10; (d) PP_PCI20.

A Tabela 1 apresenta os resultados de dureza Shore D. Houve uma pequena tendência de aumento com o teor de carga, alcançando 76 Shore D, em relação ao PP puro de 73 Shore D. O aumento não foi mais significativo já que há um teor bem maior da matriz polimérica que da carga, e também pelo fato desta ser uma medida de superfície e a superfície do compósito é naturalmente mais rica em matriz que a média do material.

Os resultados de resistência, módulo de elasticidade e deformação na ruptura em tração para as amostras de PP puro, PP_PCI5, PP_PCI10 e PP_PCI20 estão também apresentados na Tabela 1. Houve um aumento de resistência à tração de 5\% para a amostra PP_PCI5, 3,54\% para a amostra PP_PCI10 e um decréscimo de 1,54\% na amostra PP_PCI20, em ralação ao PP puro. Como os resultados não são tão expressivos, pode-se considerar que a resistência à tração do compósito desenvolvido se manteve no mínimo similar ao PP puro. ZHENG et al. 2009 [14] obtiveram resultados mais significativos em relação à resistência à tração em seu estudo com PP e resíduo de PCIs. O valor obtido para o PP puro avaliado foi de $\approx 39 \mathrm{MPa}$, que aumentou $\approx 9 \%$ para a amostra com $10 \%$ de resíduo e $\approx 15 \%$ para $20 \%$ de resíduo. As particularidades de cada estudo podem justificar essa discrepância.

Tabela 1: Dados de resistência, módulo de elasticidade e deformação na ruptura em tração, e dureza Shore D.

\begin{tabular}{c|c|c|c|c}
\hline Amostra & Dureza Shore D & $\begin{array}{c}\text { Resistencia à } \\
\text { tração (MPa) }\end{array}$ & $\begin{array}{c}\text { Módulo de } \\
\text { elasticidade (GPa) }\end{array}$ & $\begin{array}{c}\text { Deformação na } \\
\text { ruptura (\%) }\end{array}$ \\
\hline PP puro & $73 \pm 2$ & $42,40 \pm 0,37$ & $0,89 \pm 0,22$ & $24,09 \pm 1,47$ \\
\hline PP_PCI5 & $74 \pm 2$ & $44,54 \pm 0,61$ & $0,9 \pm 0,13$ & $15,98 \pm 2,00$ \\
\hline PP_PCI10 & $74 \pm 2$ & $43,90 \pm 3,13$ & $0,99 \pm 0,28$ & $10,36 \pm 2,44$ \\
\hline PP_PCI20 & $76 \pm 2$ & $41,74 \pm 0,57$ & $1,02 \pm 0,16$ & $8,86 \pm 1,90$ \\
\hline
\end{tabular}

Com o aumento do teor resíduo na matriz polimérica de compósito, os resultados de módulo de elasticidade para os compósitos mostram um aumento. O maior módulo de elasticidade é de 1,02 GPa para o compósito com 20\% de resíduo, $\approx 15 \%$ acima do valor para o PP puro. Ou seja, o material ficou mais rígido com a inclusão.

Os resultados de deformação na ruptura para as amostras estudadas foram coerentes com os resultados de módulo. Ou seja, houve uma redução progressiva da deformação na ruptura conforme aumentou o teor de resíduo na amostra. Os dados expressam uma redução significativa, pois enquanto o PP puro obteve $\approx 24 \%$ de deformação, as amostras com $5 \%, 10 \%$ e $20 \%$ de resíduo alcançaram valores de $\approx 16 \%, 10$ e $9 \%$, respectivamente. Assim, vê-se uma clara redução da ductilidade com o aumento do teor de resíduo. Resultados similares foram relatados por MUNIYANDI et al. 2013 [16] para PEAD e resíduo não-metálico de PCIs. Assim como neste estudo, os autores utilizaram granulometria mista e obtiveram decréscimo para as formulações com $10 \%$ e $20 \%$ de resíduo.

\subsection{Análise morfológica}

As imagens obtidas da fratura da secção transversal dos corpos de prova com auxílio do microscópio eletrônico de varredura (MEV) permitem observar a evolução da quantidade de resíduo adicionado às amostras e como este interage com a 
matriz. Na Figura 6a é mostrada a micrografia da fratura da amostra de PP puro. A rugosidade observada e a trinca exposta é oriunda da fratura do corpo de prova de característica um pouco plástica, como também relatado por ZHENG et al. 2009 [14].

A Figura $6 \mathrm{~b}$ apresenta a micrografia da fratura da amostra PP_PCI5. É possível observar de forma pouco expressiva a presença do resíduo disperso, que apresenta alguma interação com a matriz polimérica. Pode-se observar uma fibra oriunda do resíduo na parte superior da imagem e um vazio provocado pelo destacamento de outra fibra no momento da fratura criogênica do corpo de prova. As marcas na superfície permanecem e são similares às observadas para o PP puro.
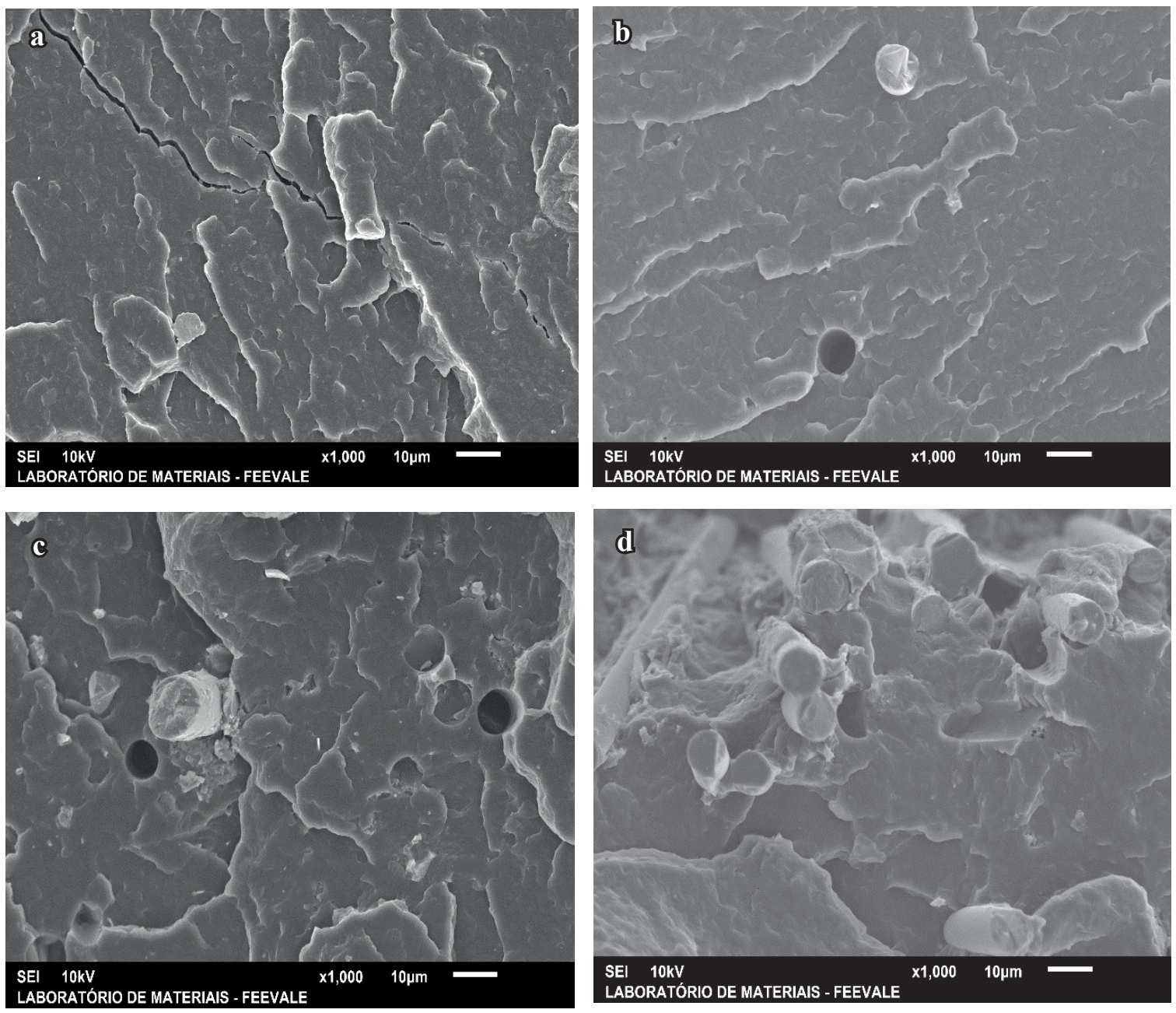

Figura 6: Micrografia da fratura das amostras de: (a) PP puro, (b) PP_PCI5, (c) PP_PCI10 e (d) PP_PCI20.

A Figura 6c apresenta a micrografia da fratura da amostra PP PCI10. A presença do resíduo já é consideravelmente maior que a anterior, sendo possível identificar novas interações. É possível observar partículas de diversos tamanhos e formas na matriz polimérica, além do aumento do número de vazios de destacamento (pull-out) e uma fibra de formato cilíndrico bem definido. MUNIYANDI et al. 2013 [16] também relataram a presença de fibras de vidro, muito presentes no resíduo de PCIs. As partículas de formato e tamanhos irregulares são descritas por SOHAIB et al. 2015 [19] como partículas/resíduos de resinas termorrígidas oriundas das PCIs. Para a amostra PP_PCI20, mostrada na Figura 6d, estas características se intensificaram, ou seja, as fibras de vidro podem ser melhor visualizadas, e houve aumento expressivo de sua presença na matriz de PP. Observa-se ainda fibras de comprimento relativamente longo, mesmo após passagem do material por processos de trituração e separação granulométrica em peneiras de pequeno diâmetro. Partículas menores são menos notadas nessa amostra e podem estar mais dispersas no restante da matriz.

Tendo em vista que estudos envolvendo resíduos de PCIs de smartphones e computadores têm sido extensivamente publicados [20-23], pode-se destacar a relevância em pesquisar outros tipos de PCIs que não recebem tanta visibilidade, mas podem também, apresentar significativo volume de descarte, bem como potencial de reaproveitamento. Neste trabalho foi utilizada uma quantidade significativa de aproximadamente $13 \mathrm{~kg}$ do mesmo modelo de PCI's empregadas em controladoras e indicadoras de temperatura de câmaras frias para obtenção do pó utilizado nos compósitos com matriz de PP. 
Além disso, o processamento contemplou as etapas de cominuição e separação granulométrica, magnética e eletrostática, permitindo, assim, uma eficiência ainda maior na separação das frações metálicas e não metálicas, bem como maior homogeneidade na composição química do pó que foi utilizado como matéria-prima do compósito.

\section{CONCLUSÕES}

Neste trabalho, avaliou-se a ação do processamento do resíduo de PCIs empregadas em controladoras e indicadoras de temperatura de câmaras frias para o desenvolvimento de compósitos com matriz de polipropileno, focando-se a análise na caracterização das propriedades físicas, mecânicas e morfológicas do compósito em relação ao PP puro e outros estudos envolvendo resíduo de PCI em matriz polimérica. Os resultados obtidos evidenciaram o potencial da utilização do pó gerado nos processos de cominuição mecânica de PCIs como carga de compósitos de matriz polimérica em substituição ao PP puro em aplicações onde as propriedades resistência à tração, rigidez e dureza sejam determinantes, mesmo para um elevado teor de resíduo (20\%) agregado, mas que não exijam tanta ductilidade, a única característica que mostrou deterioração. Assim, a prática da reciclagem desse resíduo pode aliar ganhos econômicos com a economia de matéria-prima virgem, com ganhos ambientais relacionados à disposição adequada de um resíduo nocivo para o meio-ambiente.

\section{AGRADECIMENTOS}

Os autores agradecem à revista Matéria-UFRJ, ao Laboratório de Estudos Avançados em Materiais da Universidade Feevale e também ao suporte financeiro da Coordenação de Aperfeiçoamento de Pessoal de Nível Superior (CAPES/ PROEX 23038.000341/2019-71 0491/2019) pelo apoio para confecção desse artigo.

\section{BIBLIOGRAFIA}

[1] BALDÉ, C.P., FORTI, V., GRAY, V., KUEHR, R., et al., The global e-waste monitor. Bonn: United Nations University, 2017.

[2] ADAM, A.P., GONÇALVES, J.V.RV., ROBINSON, L.C.R., et al., Recycling and Mechanical Characterization of Polymer Blends Present in Printers. Materials Research, Epub June 22, 2017.https://doi.org/10.1590/1980-5373-mr-2016-1099. n. 20, suppl. 2, pp. 202-208. 2017.

[3] LI, J., JIANG, Y., XU, Z., et al., "Eddy current separation technology for recycling printed circuit boards from crushed cell phones”, Journal Of Cleaner Production, v. 141, pp.1316-1323, Jan 2017.

[4] GHOSH, B., et al., "Waste Printed Circuit Boards recycling: an extensive assessment of current status", Journal Of Cleaner Production, v. 94, pp.5-19, Fev. 2015.

[5] KUMARA, A., HOLUSZKOA, M., ESPINOSAB, D.C.R., et al., "E-waste: An overview on generation, collection, legislation and recycling practices", Resources, Conservation And Recycling, v.1, n. 122, pp. 32-42, Jul 2017.

[6] ZENGA, X., et al., "Current status and future perspective of waste printed circuit boards recycling", Procedia Environmental Sciences, v.1, n. 16, pp. 590-597, 2012.

[7] KUMAR, V., et al., "Recycling of printed circuit boards (PCBs) to generate enriched rare metal concentrate", Journal Of Industrial And Engineering Chemistry, v.1, n. 21, pp. 805-813, Jan 2015.

[8] KOVACEVIC, T., et al., "New composites based on waste PET and non-metallic fraction from waste printed circuit boards: Mechanical and thermal properties”, Composites Part B, v.1, n. 127, pp.1-14, Oct 2017.

[9] ESTRADA-RUIZ, R.H., et al., "Separation of the metallic and non-metallic fraction from printed circuit boards employing green technology", Journal Of Hazardous Materials, v. 1, n. 311, pp. 91-99, Jul 2016.

[10] ROCCHETTI, L., AMATO, A., BEOLCHINI, F., "Printed circuit board recycling: A patent review", Journal Of Cleaner Production, v. 1, n. 178, p. 814-832, Mar 2018.

[11] LI, J., XU, Z., ZHOU, Y., et al., "Application of corona discharge and electrostatic force to separate metals and nonmetals from crushed particles of waste printed circuit boards", Journal Of Electrostatics, v. 1, n. 65, pp. 233-238, Apr 2007.

[12] ZHENG,Y., et al., "Influence of nonmetals recycled from waste printed circuit boards on flexural properties and fracture behavior of polypropylene composites”, Materials \& Design, v. 30, n. 4, pp.958-963, Apr. 2009.

[13] YANG, S., et al., "Preparation of fine fiberglass-resin powders from waste printed circuit boards by different milling methods for reinforcing polypropylene composites", Journal of Applied Polymer Science, v. 132, pp.1-8, May. 2015. 
[14] ZHENG,Y., et al., "The reuse of nonmetals recycled from waste printed circuit boards as reinforcing fillers in the polypropylene composites”, Journal of Hazardous Materials, v.1, n. 163, pp. 600-606, Apr.2009.

[15] WANG, X., et al., "PVC-based composite material containing recycled non-metallic printed circuit board (PCB) powders”, Journal Of Environmental Management, v.1, n. 91, pp. 505-510, Dec.2010.

[16] MUNIYANDI, S.K., SOHAILI, J., HASSAN, A., et al., "Mechanical, thermal, morphological and leaching properties of nonmetallic printed circuit board waste in recycled HDPE composites", Journal Of Cleaner Production, v.1, n. 57, pp. 327-334, Oct.2013.

[17] SHIN, S., MAI, v.D., LEE, D.s., et al., "Chemical Recycling of Used Printed Circuit Board Scraps: Recovery and Utilization of Organic Products”, Processes, v.7, n. 1, pp. 22-41, Jan. 2019.

[18] MAT-SHAYUTI, M.S., ABDULLAH, M.Z., MEGAT-YUSOFF, P.S.M., et al.,"Thermal properties and morphology of Polypropylene/Polycarbonate/Polypropylene-Graft-Maleic anhydride blends.”, In: Matec Web Of Conferences, Selangor, Malásia, v.1, n. 69, pp.2-6, Jun.2016.

[19] SOHAIB, Q., et al., "Comparative Analysis of Recycled PVC composites reinforced with nonmetals of printed Circuit Boards”, Research Gate, v. 45, n. 1, pp.431-436, Jun.2015.

[20] YAMANE, L.H., et al. "Recycling of WEEE: Characterization of Spent Printed Circuit Boards from Mobile Phones and Computers". Waste Management 31, pp. 2553-2558, 2011.

[21] CALGARO, C.O., et al. "Supercritical Extraction of Polymers from Printed Circuit Boards Using CO2 and Ethanol”. Journal of CO. Utilization 22, pp. 307-316, 2017.

[22] RAJAGOPAL, R.R., et al. "Sustainable Composite Panels from Non-Metallic Waste Printed Circuit Boards and Automotive Plastics". Journal of Cleaner Production 144, pp. 470-481, 2017.

[23] HOLGERSSONA, S., et al. "Analysis of The Metal Content of Small-Size Waste Electric and Electronic Equipment (WEEE) Printed Circuit Boards-Part 1: Internet Routers, Mobile Phones and Smartphones”. Resources, Conservation and Recycling 133, pp. 300-308, 2018.

\section{ORCID}

Eduardo Luis Schneider Rodrigo de Andrade Chaves Guilherme Dias Grassi Luis Carlos Robinson Sandro Campos Amico https://orcid.org/0000-0002-3938-2662 https://orcid.org/0000-0003-4144-9544 https://orcid.org/0000-0002-0052-6382 https://orcid.org/0000-0003-1109-6127 https://orcid.org/0000-0003-4873-2238 